\title{
Evaluation of Anaesthesia Technique in Emergency Caesarean Section in a Teaching Hospital of Sub Himalayan Belt - A Prospective Analysis
}

\author{
Abhishek Nautiyal ${ }^{1}$, Geeta Bhandari², Kedar Singh Shahi ${ }^{3}$, Aditya Kumar Chauhan ${ }^{4}$ \\ ${ }^{1}$ Department of Anaesthesiology, Critical Care and Pain and Palliative Medicine, Government Medical \\ College, Haldwani, Uttarakhand, India. ${ }^{2}$ Department of Anaesthesiology, Critical Care and Pain and Palliative \\ Medicine, Government Medical College, Haldwani, Uttarakhand, India. ${ }^{3}$ Department of Surgery, Government \\ Medical College, Haldwani, Uttarakhand, India. ${ }^{4}$ Department of Anaesthesiology, Critical Care and Pain and \\ Palliative Medicine, Government Medical College, Haldwani, Uttarakhand, India.
}

\section{ABSTRACT}

\section{BACKGROUND}

The number of Caesarean Deliveries has shown a rapid increase in modern obstetric practice. This study was done to evaluate the choices of anaesthetic techniques for the lower segment caesarean section (LSCS) following the patient's clinical condition and its correlation with maternal and foetal outcomes in Dr. Sushila Tiwari Govt. Hospital, Haldwani, Uttarakhand, India.

\section{METHODS}

We conducted a prospective observational study for one year. We recorded patient's age, parity, American Society of Anaesthesiologist (ASA) physical classification, chronic diseases or any other preoperative comorbid condition, caesarean indication, maternal outcomes, and foetal outcomes. We grouped the anaesthetic techniques used in LSCS as General Anaesthesia (GA) and Subarachnoid Block (SAB).

\section{RESULTS}

Out of a total of 2161 emergency caesarean sections were performed during the study period, 53 were under GA, 2096 under under SAB and in 12 cases SAB was converted to GA. Unlike previous studies, the most common indication of LSCS was previous caesarean $(40.1 \%)$. In comparison, foetal distress is the second most common cause of emergency LSCS (37.1\%). Maternal mortality was $16.9 \%(11 / 65)$ and $0.1 \%$ $(3 / 2096)$ in GA and SAB, respectively (p-value 0.001$)$. Neonatal mortality was $10.8 \%$ (7) and $2.4 \%$ (50) in GA and SAB, respectively.

\section{CONCLUSIONS}

Maternal and neonatal mortality and complications were higher in the GA group as compared to the SAB group. Also, increased incidence of violence against health care providers and rising lawsuits has led to a sense of insecurity amongst the health care providers which is evident via preference of LSCS in patients with previous history of caesarean section. This is evident from the trend that now previous LSCS is becoming most common indication of LSCS instead of foetal distress as is found in various studies done earlier.

\section{KEY WORDS}

Emergency, Caesarean Section, Maternal, Neonatal
Corresponding Author:

Geeta Bhandari,

Professor and $H O D$,

Department of Anaesthesiology,

Critical Care, Pain and Palliative Medicine, Government Medical College,

Haldwani-263139, Uttarakhand, India.

E-mail:dr.geetabhandari@gmail.com

DOI: $10.14260 /$ jemds/2020/461

How to Cite This Article:

Nautiyal A, Bhandari G, Shahi KS, et al. Evaluation of anaesthesia technique in emergency caesarean section in a teaching hospital of sub Himalayan belt- a prospective analysis. J. Evolution Med. Dent. Sci. 2020;9(30):2114-2118, DOI: 10.14260/jemds/2020/461

Submission 30-04-2020,

Peer Review 17-06-2020,

Acceptance 24-06-2020,

Published 27-07-2020.

Copyright (C) 2020 JEMDS. This is an open access article distributed under Creative Commons Attribution License [Attribution 4.0 International (CC BY 4.0)] 


\section{BACKGROUND}

In modern obstetric practice, caesarean delivery is rapidly outnumbering normal or assisted vaginal delivery. Various reasons for this change in obstetric practice primarily include a change in patient preference, increased violence against doctors, the decreased incidence of forceps or vacuumassisted delivery and an increase in the rate of institutional delivery.

Anaesthetic technique employed, and the method of delivery must be according to the clinical situation,(1) and the resources available. When time is the limiting factor, at times, general anaesthesia becomes a necessity because of its advantages like rapid induction, reliability, controllability, and avoidance of sympathectomy-induced hypotension.

Increase in the rate of caesarean delivery also corresponds to an improvement of health care delivery system over time owing to the success of government-run programs like "Janani Shishu Suraksha Yojna (JSSY)", "National Health Mission (NHM)", increased awareness among the general population regarding institutional delivery and improved perinatal screening and referral services available.

This shift towards institutionalized perinatal care has resulted in a more substantial proportion of women receiving specialized childbirth care, including caesarean sections and assisted vaginal deliveries, options for pain-controlled childbirth. Better neonatal care has improved maternal and infant well-being.(2) The National Family Health Survey-4 (2015-16) also reported that infant mortality rates reduced from 57 (NFHS-3 done in 2005-06) to 41 per 1,000 live births. $(3,4)$ The NFHS-4 also reported an increasing uptake of antenatal services compared to NFHS-3.

Still, there is a minor increase in labour trails and instrument-assisted vaginal delivery as compared to a more substantial increase in the number of caesarean deliveries. World Health Organization (WHO) suggest that although the caesarean section is a safe method of delivery. If the caesarean delivery rate is above $15 \%$, the risk of manifestation of a significant public health problem for mothers and children also increases.

Due to such an increase in caesarean delivery, there has been a constant increase in the involvement of anaesthesiologist in obstetric cases. Thus, there is an evolution of obstetric anaesthesia as a sub-speciality within anaesthesia that has to simultaneously monitor the health of the pregnant mother and the growing foetus or baby.

Our hospital located in the sub-Himalayan region caters to patients from a wide range of ethnic and linguistic groups. Many of these patients are unbooked and not investigated and often presents in labour. Hence, the purpose study is to quantify the data of all the childbirth and to evaluate the choices of anaesthetic technique for emergency Caesarean Section per patient's clinical conditions and its correlation with maternal and foetal outcomes.

\section{METHODS}

The study was approved by the appropriate Institutional Ethical Committee (IEC), it was an observational study, written informed consent was obtained for surgery and anaesthesia as per institutional protocol.

It was an observational prospective clinical study which included all cases of emergency caesarean section for one year (Jan 2018-Dec 2018) after getting permission from institutional ethical committee except the caesarean section conducted for Intrauterine Death (IUD).

The following maternal and neonatal details were recorded for all the cases during the study period

- Patient's age, parity, ASA physical classification, chronic diseases or any other preoperative comorbid condition and caesarean indication.

- Maternal outcome as recorded as morbidity which includes any haemorrhage, intraoperative event, complication related to anaesthesia or surgery, Intensive care unit (ICU) admission, the complication in maternity ward (hypoxia, hypotension, seizure) duration of anaesthesia and surgery and maternal mortality.

- Foetal outcome as APGAR score, length of Neonatal intensive care unit (NICU) stays for newborn and requirement of oxygen therapy or positive pressure ventilation and stillbirth.

- While recording, the anaesthetic techniques used in lower segment caesarean section (LSCS) were grouped as General Anaesthesia (GA) and Subarachnoid Block (SAB).

\section{Statistical Analysis}

Data were analyzed and statistically evaluated using SPSS-20 version. Quantitative data were expressed in mean, standard deviation, while qualitative data expressed in percentage comparison of quantitative data between two group done by Student t-test. The chi-square test tested statistical differences between the proportions. ' $P$ ' value of less than 0.05 was considered statistically significant.

\section{RESULTS}

During the study period, 2161 emergency caesarean sections were performed, which includes $2.4 \%$ (53) under general anaesthesia, $96.9 \%$ (2096) performed under regional anaesthesia and in $0.5 \%$ (12) cases regional was converted to general anaesthesia due to surgical causes. Elective caesarean deliveries were 72 . The total number of vaginal deliveries during this period was 1987. Thus, the total number of deliveries during the study period were 4220 . Thus, caesarean deliveries were $52.91 \%$ of all delivery during this period in our hospital.

In our study, we found that the maximum number of subjects in both (GA and SAB) the group were of 21 - 30 years of age (Table 1). The maximum number of cases in both groups had a history of previous LSCS $29.2 \%$ (19) and $40.4 \%$ (847) for GA and SAB group. In comparison, foetal distress is the second most common cause of emergency LSCS, forming 24.6\% (16) and $37.5 \%$ (786) of total cases in GA and $\mathrm{SAB}$ group, respectively.

The overall rate of ICU admission was $76.9 \%$ (50) and $6.9 \%$ (144) in GA and SAB group, respectively, this difference was statistically significant (table 2). Mean duration of ICU stay in GA group was $4.12 \pm 2.14$ days and in SAB group was $2.78 \pm$ 
1.43 days (p-value 0.001) this difference is statistically significant.

\begin{tabular}{|ccccc|}
\hline Age Group & GA & SAB & Total & \multirow{2}{*}{ P Value* } \\
No. (\%) & No. (\%) & No. (\%) & \\
$21-30$ years & $7(10.8)$ & $161(7.7)$ & $168(7.8)$ & \\
$>31$ years & $48(73.8)$ & $1730(82.5)$ & $1778(82.3)$ & 0.19 \\
Total & $10(15.4)$ & $205(9.8)$ & $215(9.9)$ & \\
\hline \multicolumn{4}{|c|}{ Table 1. Comparison of Age Group in both the Study Groups } \\
\hline
\end{tabular}

\begin{tabular}{|cccc|}
\hline Indication of LSCS & GA & SAB & Total \\
& No. (\%) & No. (\%) & No. (\%) \\
Foetal Distress & $16(24.6)$ & $786(37.5)$ & $802(37.1)$ \\
Breech & $0(0.0)$ & $119(5.7)$ & $119(5.5)$ \\
Previous LSCS & $19(29.2)$ & $847(40.4)$ & $866(40.1)$ \\
Twin Pregnancy & $2(3.1)$ & $27(1.3)$ & $29(1.3)$ \\
Severe Oligo & $1(1.5)$ & $65(3.1)$ & $66(3.1)$ \\
APH & $11(16.9)$ & $26(1.2)$ & $37(1.7)$ \\
Eclampsia & $12(18.5)$ & $3(0.1)$ & $15(0.7)$ \\
CPD & $2(3.1)$ & $73(3.5)$ & $75(3.5)$ \\
NPOL & $2(3.1)$ & $129(6.2)$ & $131(6.1)$ \\
Others & $0(0.0)$ & $21(1.0)$ & $21(1.0$ \\
\hline Table 2. Comparison of Indication between GA and SAB Group \\
\hline \multicolumn{4}{r}{} \\
\hline \multicolumn{4}{r}{}
\end{tabular}

\begin{tabular}{|cccc|}
\hline $\begin{array}{c}\text { Maternal } \\
\text { Complication }\end{array}$ & $\begin{array}{c}\text { GA } \\
\text { No. (\%) }\end{array}$ & $\begin{array}{c}\text { SAB } \\
\text { No. (\%) }\end{array}$ & $\begin{array}{c}\text { Total } \\
\text { No. (\%) }\end{array}$ \\
Respiratory Distress & $5(7.7)$ & $5(0.2)$ & $10(0.5)$ \\
Anaemia & $1(1.5)$ & $7(0.3)$ & $8(0.4)$ \\
Shock & $9(13.8)$ & $10(0.5)$ & $19(0.9)$ \\
ARF & $1(1.5)$ & $0(0.0)$ & $1(0.0)$ \\
Cardiac & $0(0.0)$ & $1(0.0)$ & $1(0.0)$ \\
Sepsis & $0(0.0)$ & $1(0.0)$ & $1(0.0)$ \\
HTN & $0(0.0)$ & $4(0.2)$ & $4(0.2)$ \\
Seizure & $0(0.0)$ & $3(0.1)$ & $3(0.1)$ \\
None & $49(75.4)$ & $2065(98.5)$ & $2114(97.8)$ \\
\hline \multicolumn{4}{r}{ Table 3. Comparison of Maternal Complications } \\
between GA and SAB Group \\
\hline
\end{tabular}

\begin{tabular}{|ccccc|}
\hline Maternal & GA & SA & Total & P Value* \\
Mortality & No. (\%) & No. (\%) & No. (\%) & \\
Present & $11(16.9)$ & $3(0.1)$ & $14(0.6)$ & 0.001 \\
Absent & $54(83.1)$ & $2093(99.9)$ & $2147(99.4)$ & \\
Total & $65(100.0)$ & $2096(100.0)$ & $2161(100.0)$ \\
\hline \multicolumn{5}{c}{ Table 4. Comparison of Maternal Mortality } \\
between GA and SAB Group
\end{tabular}

\begin{tabular}{|cccc|}
\hline Variable & $\begin{array}{c}\text { GA } \\
\text { Mean } \pm \text { SD }\end{array}$ & $\begin{array}{c}\text { SA } \\
\text { Mean } \pm \text { SD }\end{array}$ & P Value* $^{*}$ \\
APGAR at 1 & $6.18 \pm 1.90$ & $7.24 \pm 1.01$ & 0.001 \\
APGAR at 5 & $7.69 \pm 1.94$ & $8.30 \pm 0.67$ & 0.001 \\
\hline \multicolumn{4}{|c|}{ Table 5. Comparison of Newborn APGAR Score } \\
at 1 and 5 min between GA and SAB Group & \\
\hline
\end{tabular}

The most common cause of ICU admission in GA group was post-op mechanical ventilation $31(47.6 \%)$ and in SAB group was post-op monitoring in 121 (5.8\%) cases.

The most common maternal complication in both GA and SAB group was shock due to obstetric haemorrhage, which accounts for complication in $13.8 \%$ and $0.5 \%$ of total cases in GA and SAB group, respectively. Respiratory distress was the second most common complication in GA group seen in $7.7 \%$ of total cases but in SAB group second most common complication was found to be anaemia in the postoperative period $(0.3 \%)$ followed by respiratory distress in $0.2 \%$ of total cases (table 3).

The main indication of GA was failed SAB in $19(29.2 \%)$ patients while the continuum of severe preeclampsia, HELLP (haemolysis, elevated liver enzymes, and low platelets) syndrome and eclampsia in $18(27.6 \%)$ patients and in $9(13.8 \%)$ patients GA was administered on maternal request

There were 14 maternal deaths amongst study subjects, maternal mortality was found to be 0.65 per 100 cases. $16.9 \%$ $(11 / 65)$ and $0.1 \%(3 / 2096)$ in GA and $\mathrm{SAB}$, respectively (Table 4).
Mean APGAR score at $1 \mathrm{~min}$ and $5 \mathrm{~min}$ was $6.18 \pm 1.90$ and $7.69 \pm 1.94$ respectively in the GA group and $7.24 \pm 1.01$ and $8.30 \pm 0.67$ in SAB group (table 5).

The most common cause for NICU admission in GA group was respiratory distress syndrome (RDS) in 15.4\% newborn of GA group in comparison to 3.9\% newborn of SAB group, second most common in GA group was neonatal jaundice in $7.7 \%$ newborn.

Neonatal jaundice was the most common diagnosis amongst neonates in SAB group, but the incidence rate (5.6\%) was lesser than the GA group.

Total 20 (30.8\%) out of 65 newborn from GA group required NICU admission and 246 (11.7\%) out of 2096 newborn from SAB group required NICU admission (table 6). The mean duration of NICU stays in GA group was $2.85 \pm 1.57$, while in SAB group it was found to be $3.43 \pm 2.51$. Still, the pvalue was 0.31 , i.e. the difference in NICU remains between two groups was not statistically significant. Neonatal mortality was $10.8 \%$ (7), and $2.4 \%$ (50) in GA and SAB group, respectively ( $p$-value 0.001), and this difference was statistically significant.

\section{DISCUSSION}

We conducted an observational study for one year, and the caesarean delivery rate was $52.91 \%$ during this period in our hospital. Worldwide, also caesarean section (CS) rates have shown increasing trend during the last few decades, (5) resulting in an overall CS rate to be nearly $26 \%$ in 2010.(6) There is no guideline about optimal CS rate.(7) The most widely accepted recommendation to date is the one from WHO, 1985, stating that the CS rate should not exceed $15 \%$.8) In 2009 WHO revised their guidelines, still recommending a CS rate between 5 and $15 \%$.9) A. Palanisamy et al.(10) found percentage of caesarean deliveries varied from $23.7 \%$ to $31.5 \%$, with a progressive increase occurring in each subsequent year between 2001 to 2005 .

Increasing maternal age is associated with specific adverse outcomes. In our study, we found that the mean age for GA is $26.94 \pm 4.94$ years and mean age for $\mathrm{SAB}$ was $25.51 \pm 3.93$ years. In a study done by Thangaswamy et al.(11) mean maternal age was $25.64 \pm 4.0$ years in Indian population. The result observed in our study is similar to that found in the above study.

In our study, the majority number of cases belonged to the age group of 21 to 30 year of age, which constituted a total of 1778 caesarean delivery which is $82.3 \%$ of total caesarean delivery. In a study by Jeremy Juang et al.(12) $76.25 \%$ of patients were between 21 and 35 years of age.

Out of 2161 caesarean deliveries occurring during the study period, 854 (39.5\%) were primi, 812 (37.6\%) were gravid of second order while $495(22.9 \%)$ were multigravida. In the study by A. Palanisamy et al.(10) they found that $41.67 \%$ of the patients were primigravida while $25.00 \%$ of the patients were in their second pregnancy, results in this study were consistent with our study.

In our study, maximum patients in GA group were of ASA III E $46.2 \%$ (30), 30.8\% (20) of ASA II E and 23.1\% (20) were of ASA grade IV $E$ and in SAB group maximum patients belong to ASA II E 90.4\% (1894), 9.5\% (199) ASA III E while 0.1\% (3) 
to ASA grade IV E. Jeremy Juang et al,(12) in their study observed $85.98 \%$ cases of SAB group were in ASA II E, and $14.02 \%$ of SAB cases were of ASA III E and ASA IV. In contrast, in GA group, $71.21 \%$ of study subject were ASA II, and $28.79 \%$ were in high-risk ASA group.

Maximum number of cases in both group was history of previous LSCS $29.2 \%$ (19) and 37.5\% (847) for GA and SAB group respectively, while foetal distress is the second most common cause of emergency LSCS forming $24.6 \%$ (16) and $37.5 \%$ (786) of total cases in GA and SAB group respectively. In a study done by Kan et al,(13) the emergency GA group, operative delivery was indicated based on a non-reassuring foetal status $(32.7 \%)$, failure of labour to progress $(22.0 \%)$ and established labour in parturients with previous uterine surgery (14.7\%). In a study done by Thangaswamy et al,(11) out of 114 emergency LSCS, 56 (49.12\%) had foetal distress as an indication.

In our study, on the contrary to other studies, we found previous LSCS to be the most common indication, the previous caesarean section does not necessarily require caesarean delivery in subsequent pregnancies. However, in our study, we have observed it as the most common indication for emergency caesarean section, the sense of insecurity amongst obstetricians and mothers seems to be responsible for repeated caesarean deliveries (14). Vaginal birth after previous caesarean delivery is often considered risky, and there is a risk of rare but serious adverse outcomes (increased rate of perinatal deaths and hypoxic brain damage). In contrast, with repeat caesarean, the risks are more frequent but less severe (15).

The Apgar score system at 1 minute and 5 minute gives us information about the severity and prognosis of asphyxia. The 1-minute score correlates with survival, while the 5-minute score is related to neurologic outcome.(16) In our study, we have found that in GA group, mean APGAR scores at 1 min and 5 min was $6.18 \pm 1.90$ and $7.69 \pm 1.94$ respectively while in SAB group mean APGAR score at $1 \mathrm{~min}$ and $5 \mathrm{~min}$ was to be $7.24 \pm 1.01$ and $8.30 \pm 0.67$ respectively. Sri Wahjoeningsih et al.(17) in their study found that Apgar score at 1 minute was $50.00 \%$ above the median, while at 5 minutes was $65.15 \%$ above the median, in SAB group. The result observed in our study is similar to that of the study mentioned above.

Also, in the past few years, the mode of delivery has increasingly become a matter of risk-orientated, defensive obstetric practice.(18) In the past few years, there have been various cases of assault against medical personnel; this fear of litigation and assault has mostly affected the decision making on the part of treating doctors also. Thus, we can see fewer trials for a vaginal delivery after previous LSCS or uterine surgery like myomectomy.

In our study, the primary indication of GA was to be failed $\mathrm{SAB}$ in $19(0.8 \%$ of total $\mathrm{SAB})$. In contrast, the continuum of severe preeclampsia, HELLP (haemolysis, elevated liver enzymes, and low platelets) syndrome and eclampsia in 18 (27.6\%) patients, GA given on maternal request to $9(13.8 \%)$ patient. In the study done by A. Palanisamy et al.(10) main indication for GA was a perceived lack of time for neuraxial anaesthesia, accounting for at least $50 \%$ of the cases. Maternal contraindications to neuraxial anaesthesia were the next major factor, comprising $28.6 \%$ of all cases. We found that the overall rate of ICU admission is 8.98\% (194). 76.9\% (50) and $6.9 \%$ (144) in GA and SAB, respectively. Mean duration of ICU stay in GA group was found to be $4.12 \pm 2.14$ days while mean duration of ICU remains in SAB group was $2.78 \pm 1.43$ days ( $p$ value 0.001 ) and we found this difference to be statistically significant.

The most common cause of ICU admission in GA group was post-op mechanical ventilation $31(47.6 \%)$ and in SAB group was post-op monitoring in 121 (5.8\%) cases.

We found that the most common maternal complication in both GA and SAB group in shock due to obstetric haemorrhage, which accounts for complication in $13.8 \%$ and $0.5 \%$ of total cases in GA and SAB group respectively. The second most common complication in GA group was respiratory distress in $7.7 \%$ of total cases but in RA group second most common complication was found to be anaemia in the postoperative period $(0.3 \%)$ followed by respiratory distress in $0.2 \%$ of total cases. In a study done by Minal Harde et al,(19) post-anaesthesia intensive care unit admission rate was $2.8 \%$. Obstetric indications $(67.2 \%)$ were the most common cause of admission to PACU. Among the obstetric indications, haemorrhage $(36.1 \%)$ was a statistically significant indication, followed by the hypertensive disorder of pregnancy (29.5\%).

In our study we found that in GA group, maternal complications were present in $16(24.6 \%)$ out of 65 patients and in SAB group maternal complications were present in 31 $(1.5 \%)$ out of 2096 patients. The most common maternal complication in both GA and SAB group was shock due to obstetric haemorrhage. It accounts for complication in $13.8 \%$ and $0.5 \%$ of the total cases in GA and SAB group, respectively.

We found that with 14 maternal deaths amongst 2161 emergency LSCS during study period maternal mortality was found to be 0.65 per 100 cases, maternal mortality was $16.9 \%$ $(11 / 65)$ and $0.1 \%(3 / 2096)$ in GA and SAB respectively (pvalue 0.001 ) and this difference was statistically significant.

The most common reason for NICU admission in GA group was RDS in $15.4 \%$ newborn delivered under GA comparing to $3.9 \%$ newborn delivered under SAB, second most common diagnosis in newborn delivered under GA is neonatal jaundice which in $7.7 \%$ of the newborn in GA group.

In comparison, neonatal jaundice was the most common diagnosis amongst neonates in SAB group, but the incidence rate $(5.6 \%)$ was lesser than the GA group. Neonatal mortality was $10.8 \%$ (7), and $2.4 \%$ (50) in GA and SAB, respectively and this difference was statistically significant. In a study done by Thangaswamy et al.(11) the main indication for NICU admission was respiratory distress in 27 (79.4\%) neonates, and the other reasons include prematurity, poor muscle tone and screening for infection. The mean duration of NICU stay $(2.08 \pm 0.82$ days in group GA vs $1.8 \pm 1.03$ days in group SA, $\mathrm{P}=0.41$ ) and neonatal mortality [3 (4.5\%) in group GA vs $2(4.3 \%)$ in group $\mathrm{SA}, \mathrm{P}=0.32$ ) were comparable between the two groups.

Financial or Other Competing Interests: None.

\section{CONCLUSIONS}

GA group is associated with significantly higher maternal and foetal complications, rate of ICU admission, maternal and neonatal mortality, lower APGAR score at 1 and 5 min than in SAB group. Caesarean rates in India has risen alarmingly like an epidemic, and this is evident from a trend that now previous 
LSCS is becoming the most common indication for LSCS in our study instead of foetal distress which is the most common indication according to various other studies done earlier. Increased violence against doctors, unethical private practices for profit are some of the reasons for caesarean sections for non-medical reasons. Factors associated with violence against doctors in India include a long waiting period, non-availability of crucial investigations, delay in referral, unhygienic and extremely crowded condition in the emergency and other wards.

A sound audit system is required to monitor the CS in both private and public health care institutions. The government should formulate stringent laws against anybody who harms the doctor and vandalizes the hospital. We recommend a multicentric study involving hospitals from a different region to ascertain this fact.

\section{REFERENCES}

[1] Al-Mufti R, McCarthy A, Fisk NM. Survey of obstetricians' personal preference and discretionary practice. Eur J Obstet Gynecol Reprod Biol 1997;73(1):1-4.

[2] Verma V, Nath DC. Prevalence of the caesarean section in India. 2007:1-17.

[3] National Family Health Survey 4, India. http://www.rchiips.org/NFHS/pdf/NFHS4/India.pdf.

[4] National Institution for Transforming India Aayog. National Institution for Transforming India. http: //www. niti.gov.in/ content/ maternal-mortality-ratio-mmr100000-livebirths.

[5] Kupari M, Talola N, Luukkaala T, et al. Does an increased caesarean section rate improve neonatal outcome in term pregnancies? Arch Gynecol Obstet 2016;294(1):41-6.

[6] Prakash KC, Neupane S. Caesarean deliveries among Nepalese mothers: changes over time 2001-2011 and determinants. Arch Gynecol Obstet 2014;289(2):421-7.

[7] Karkee R, Lee AH, Khanal V, et al. Obstetric complications and caesarean delivery in Nepal. Int J Gynaecol Obstet 2014;125(1):33-6.

[8] Irani M, Deering S. Challenges affecting access to caesarean delivery and strategies to overcome them in low-income countries. Int J Gynaecol Obstet 2015;131(1):30-4.

[9] World Health Organization. Monitoring emergency obstetric care. 2009. http://whqlibdoc.who.int/publications/2009/97892415 47734_eng.pdf?ua=1.

[10] Palanisamy A, Mitani AA, Tsen LC. General anesthesia for caesarean delivery at a tertiary care hospital from 2000 to 2005; a retrospective analysis and 10 year update. Int J Obstet Anesth 2011;20(1):10-6.

[11] Thangaswamy CR, Kundra P, Velayudhan S, et al. Influence of anaesthetic technique on maternal and foetal outcome in category 1 caesarean sections - A prospective single-centre observational study. Indian J Anaesth 2018;62:844-50.

[12] Juang J, Gabriel RA, Dutton RP, et al. Choice of anesthesia for caesarean delivery: an analysis of the national anesthesia clinical outcomes registry. Anesth Analg 2017;124(6):1914-7.

[13] Kan RK, Lew E, Yeo SW, et al. General anesthesia for caesarean section in a Singapore maternity hospital: a retrospective survey. Int J Obstet Anesth 2004;13(4):2216.

[14] Patel RM, Jain L. Delivery after previous caesarean: shortterm perinatal outcomes. Semin Perinatol 2010;34(4):272-80.

[15] Harris LH. Counselling women about choice. Best Pract Res Clin Obstet Gynaecol 2001;15(1):93-107.

[16] Practice guidelines for obstetric anesthesia: an updated report by the American Society of Anesthesiologists Task Force on Obstetric Anesthesia and the Society for Obstetric Anesthesia and Perinatology. Anesthesiology 2016;124(2):270-300.

[17] Wahjoeningsih S, Witjaksono W. Evaluation of anaesthesia methods in caesarean section for foetal distress. Malays J Med Sci 2007;14(2):41-6.

[18] Minkoff H, Paltrow LM. Melissa rowland and the rights of pregnant women. Obstet Gynecol 2004;104(6):1234-6.

[19] Harde M, Dave S, Wagh S, et al. Prospective evaluation of maternal morbidity and mortality in post-caesarean section patients admitted to postanesthesia intensive care unit. Anesthesiol Clin Pharmacol 2014;30(4):508-13. 